\title{
Zwei Jahrzehnte Deutsche Atemwegsliga
}

\author{
Die Deutsche Atemwegsliga feierte letztes Jahr ihr zwanzig- \\ jähriges Bestehen - ein Grund zum Rückblick auf Geleistetes, \\ aber auch für den Blick nach vorne.
}

C. hronische Atemwegserkrankungen stellen nach wie vor ein großes medizinisches, soziales und volkswirtschaftliches Problem dar. Die Arbeit der vor zwei Jahrzehnten gegründeten Deutschen Atemwegsliga ist deshalb noch längst nicht überflüssig. Mit gezielten Informationen für Fachkreise und Patienten kämpft die Liga weiter gegen Unwissenheit und Unterversorgung auf dem Gebiet der Atemwegserkrankungen.

\section{Angesprochen werden Ärzte, ...}

Wie H. Worth (Fürth), der Vorsitzende der Deutschen Atemwegsliga, ausführte, sollen die eingeschlagenen und bewährten Wege weiter verfolgt und neue, zukunftsorientierte Konzepte erarbeitet werden. So wird auf Ärzteebene die Reihe der Expertenempfehlungen zügig fortgesetzt - geplant sind Themen wie Sport und körperliches Training bei obstruktiven Atemwegserkrankungen oder Umwelteinflüsse bei Atemwegs- und Lungenkrankheiten. Weiter ausgebaut wird das sich großen Zulaufs erfreuende Angebot von Trainerseminaren für Ärzte zur Schulung erwachsener Asthmatiker. Hauptziel dieser Seminare ist das Einüben von patientengerechtem Lernverhalten auf der Basis strukturierter und evaluierter Schulungsprogramme.

\section{... Patienten und Interessierte ...}

Zunehmend größere Bedeutung erhalten aber auch Aktionen, die sich direkt an die Öffentlichkeit und die Betroffenen wenden. So sollen im Rahmen von Präventionsprogrammen Kinder in den Grundschulklassen z.B. über die Gefahren des Nikotinkonsums aufgeklärt werden. Der 1998 inaugurierte Deutsche Lungentag, ausgerichtet zusammen mit anderen Verbänden und Fachgesellschaften, wendet sich mit seinen breit gefächerten Informationen zur Präven- tion und Therapie sowohl an die allgemeine Öffentlichkeit wie auch Lungenpatienten im Besonderen.

Wichtiger aber noch sind konkrete Hilfsangebote für Patienten: Bekannt und beliebt sind die Schulungen asthmakranker Kinder und Erwachsener. Angeboten werden darüber hinaus aber auch Programme für das körperliche Training Lungenkranker, dessen lebensqualitätssteigernde und gesundheitsfördernde Wirkung z.B. für Asthmapatienten wissenschaftlich gesichert ist.

\section{... und Selbsthilfegruppen}

Auf diesem Gebiet besteht noch Nachholbedarf, denn den immerhin etwa 4000 Koronarsportgruppen stehen heute nur etwa 150 Lungensportgruppen gegenüber. Deshalb wurde in Kooperation mit anderen Fachverbänden die „Arbeitsgemeinschaft Lungensport" ins Leben gerufen. Ihr Ziel: die Förderung und Gründung weiterer ambulanter Lungensportgruppen.

\section{Die Deutsche Atemwegsliga stellt sich vor}

Hauptziel der Aktivitäten der Deutschen Atemwegsliga ist, Patienten mit Atemwegsund Lungenkrankheiten durch adäquate, frühzeitige Diagnostik und eine optimale Therapie ein möglichst hohes Maß an Lebensqualität trotz ihrer meist chronischen Erkrankung zu vermitteln. Angesprochen werden daher sowohl die betreuenden Ärzte wie auch die Atemwegspatienten direkt.

Dadurch ergeben sich die verschiedenen Arbeitsschwerpunkte der Liga:

- Die Veröffentlichung von Empfehlungen zu Prävention, Diagnostik und Therapie unter Einschluß von Rehabilitationsmaßnahmen für die Patienten,

- die Fortbildung von Ärzten,

$\checkmark$ die Information von Patienten und der Öffentlichkeit,

> die Unterstützung von Programmen zur Prophylaxe und Früherkennung von Atemwegserkrankungen,

$\checkmark$ die Förderung der pneumologischen Forschung sowie

- die Förderung der Qualitätssicherung in Diagnostik und Therapie.

Kontaktadresse: Deutsche Atemwegsliga e.V., Burgstraße 12,

33175 Bad Lippspringe, Tel.: 052 52/393 36 15, Fax: 052 52/39336 15,

E-Mail: Atemwegsliga.Lippspringe@t-online.de 